\title{
An Existence Result for Neutral Delay Integrodifferential Equations with Fractional Order and Nonlocal Conditions
}

\author{
Fang Li ${ }^{1}$ and Gaston M. N'Guérékata ${ }^{2}$ \\ ${ }^{1}$ School of Mathematics, Yunnan Normal University, Kunming 650092, China \\ ${ }^{2}$ Department of Mathematics, Morgan State University, 1700 East Cold Spring Lane, \\ Baltimore, MD 21251, USA \\ Correspondence should be addressed to Fang Li, fangli860@gmail.com \\ Received 27 July 2011; Revised 14 September 2011; Accepted 14 September 2011 \\ Academic Editor: Svatoslav Staněk
}

Copyright (C) 2011 F. Li and G. M. N'Guérékata. This is an open access article distributed under the Creative Commons Attribution License, which permits unrestricted use, distribution, and reproduction in any medium, provided the original work is properly cited.

\begin{abstract}
We study the existence of mild solutions of a class of neutral delay integrodifferential equations with fractional order and nonlocal conditions in a Banach space $X$. An existence result on the mild solution is obtained by using the theory of the measures of noncompactness and the theory of condensing maps. Two examples are given to illustrate the existence theorem.
\end{abstract}

\section{Introduction}

Differential and integrodifferential equations of the fractional order are playing an increasingly important role in engineering, physics, and other fields of science, such as the fractal theory and the diffusion in porous media, electrolysis chemical, fractional biological neurons, condensate physics, statistical mechanics, so they attract the attention of many researchers (see, e.g., [1-12] and the references therein).

Moreover, the Cauchy problem for various delay equations in Banach spaces has been receiving more and more attention during the past decades (see, e.g., $[3,4,6,7,9,13,14]$ ).

This paper is concerned with an existence result for nonlocal neutral delay fractional integrodifferential equations in a separable Banach space $X$ :

$$
\begin{gathered}
D^{q}\left(x(t)-h\left(t, x_{t}\right)\right)=A\left(x(t)-h\left(t, x_{t}\right)\right)+\int_{0}^{t} K(t, s) f\left(s, x(s), x_{s}\right) d s, \quad t \in[0, T], \\
x(t)=g(x)(t)+\phi(t), \quad t \in[-r, 0],
\end{gathered}
$$

where $T>0,0<q<1,0<r<\infty$. The fractional derivative is understood here in the Caputo sense. $A$ is the infinitesimal generator of an analytic semigroup $S(\cdot)$ of uniformly 
bounded linear operators on $X$, that is, there exists $M \geq 1$ such that $\|S(t)\| \leq M$. Here $h$ : $[0, T] \times C([-r, 0], X) \rightarrow X, f:[0, T] \times X \times C([-r, 0], X) \rightarrow X, K: D \rightarrow \mathbf{R}(D=\{(t, s) \in$ $[0, T] \times[0, T]: t \geq s\}), g: C([-r, 0], X) \rightarrow C([-r, 0], X), \phi \in C([-r, 0], X)$, where $C([a, b], X)$ denotes the space of all continuous functions from $[a, b]$ to $X$.

For any continuous function $x$ defined on the interval $[-r, T]$ and any $t \in[0, T]$, we denote by $x_{t}$ the element of $C([-r, 0], X)$ defined by $x_{t}(\theta)=x(t+\theta)$ for $\theta \in[-r, 0]$.

The nonlocal condition can be applied in physics with better effect than that of the classical initial condition. There have been many significant developments in the study of nonlocal Cauchy problems (see, e.g., [10, 15-19] and references cited there in). To the authors' knowledge, few papers can be found in the literature for the solvability of the fractional order delay integrodifferential equations of neutral type with nonlocal conditions.

In this paper, motivated by above works, we study the neutral delay fractional integrodifferential equations with nonlocal condition (1.1) in a separable Banach space $X$ and obtain the existence theorem based on a special measure of noncompactness without the assumptions that the nonlinearity $f$ satisfies a Lipschitz type condition and the semigroup $\{S(t)\}_{t \geq 0}$ generated by $A$ is compact. Two examples are given to show the applications of the abstract result.

\section{Preliminaries}

Throughout this paper, we denote by $X$ a separable Banach space with norm $\|\cdot\|$, by $L(X)$ the Banach space of all linear and bounded operators on $X$, and by $C([a, b], X)$ the space of all $X$-valued continuous functions on $[a, b]$ with the supremum norm as follows:

$$
\|x\|_{[a, b]}=\|x\|_{C([a, b], X)}=\sup \{\|x(t)\|: t \in[a, b]\}, \quad \text { for any } x \in C([a, b], X) .
$$

Moreover, we abbreviate $\|u\|_{L^{1}\left([0, T], \mathbf{R}^{+}\right)}$with $\|u\|_{L^{1}}$, for any $u \in L^{1}\left([0, T], \mathbf{R}^{+}\right)$.

We will need the following facts from the theory of measures of noncompactness and condensing maps (see, e.g., $[20,21])$.

Definition 2.1. Let $E$ be a Banach space and $(\mathcal{A}, \geq)$ a partially ordered set. A function $\beta$ : $P(E) \rightarrow \mathcal{A}$ is called a measure of noncompactness (MNC) in $E$ if

$$
\beta(\overline{\mathrm{co}}(\Omega))=\beta(\Omega) \quad \text { for every } \Omega \in P(E),
$$

where $P(E)$ denotes the class of all nonempty subsets of $E$.

A MNC $\beta$ is called:

(i) monotone, if $\Omega_{0}, \Omega_{1} \in P(E), \Omega_{0} \subset \Omega_{1}$ implies $\beta\left(\Omega_{0}\right) \leq \beta\left(\Omega_{1}\right)$;

(ii) nonsingular, if $\beta(\{a\} \cup \Omega)=\beta(\Omega)$ for every $a \in E, \Omega \in P(E)$;

(iii) invariant with respect to union with compact sets, if $\beta(\{D\} \cup \Omega)=\beta(\Omega)$ for every relatively compact set $D \subset E, \Omega \in P(E)$.

If $\mathcal{A}$ is a cone in a normed space, we say that the MNC $\beta$ is

(iv) algebraically semiadditive, if $\beta\left(\Omega_{0}+\Omega_{1}\right) \leq \beta\left(\Omega_{0}\right)+\beta\left(\Omega_{1}\right)$ for each $\Omega_{0}, \Omega_{1} \in P(E)$;

(v) regular, if $\beta(\Omega)=0$ is equivalent to the relative compactness of $\Omega$;

(vi) real, if $\mathbb{A}$ is $[0,+\infty)$ with the natural order.

As an example of the MNC possessing all these properties, we may consider the Hausdorff MNC

$$
\chi(\Omega)=\inf \{\varepsilon>0: \Omega \text { has a finite } \varepsilon \text {-net }\} .
$$


Now, let $G:[0, h] \rightarrow P(E)$ be a multifunction. It is called:

(i) integrable, if it admits a Bochner integrable selection $g:[0, h] \rightarrow E, g(t) \in G(t)$ for a.e. $t \in[0, h]$;

(ii) integrably bounded, if there exists a function $\vartheta \in L^{1}([0, h], E)$ such that

$$
\|G(t)\|:=\sup \{\|g\|: g \in G(t)\} \leq \vartheta(t), \quad \text { a.e. } t \in[0, h] .
$$

We present the following assertion about $\mathcal{X}$-estimates for a multivalued integral [21, Theorem 4.2.3].

Proposition 2.2. For an integrable, integrably bounded multifunction $G:[0, h] \rightarrow P(X)$, where $X$ is a separable Banach space, let

$$
X(G(t)) \leq q(t), \quad \text { for a.e. } t \in[0, h],
$$

where $q \in L_{+}^{1}([0, h])$. Then, $X\left(\int_{0}^{t} G(s) d s\right) \leq \int_{0}^{t} q(s) d s$ for all $t \in[0, h]$.

Let $E$ be a Banach space, and $\beta$ a monotone nonsingular MNC in $E$.

Definition 2.3. A continuous map $\mathfrak{F}: Y \subseteq E \rightarrow E$ is called condensing with respect to a MNC $\beta$ (or $\beta$-condensing) if, for every bounded set $\Omega \subseteq Y$ which is not relatively compact, we have

$$
\beta(\mathfrak{F}(\Omega)) \nsupseteq \beta(\Omega) .
$$

The following fixed point principle (see, e.g., $[20,21]$ ) will be used later.

Theorem 2.4. Let $\mathfrak{M}$ be a bounded convex closed subset of $E$ and $\mathfrak{F}: \mathfrak{M} \rightarrow \mathfrak{M}$ a $\beta$-condensing map. Then, Fix $\mathfrak{F}=\{x: x=\mathfrak{F}(x)\}$ is nonempty.

Theorem 2.5. Let $V \subset E$ be a bounded open neighborhood of zero and $\mathfrak{F}: \bar{V} \rightarrow E$ a $\beta$-condensing map satisfying the boundary condition

$$
x \neq \lambda \mathfrak{F}(x)
$$

for all $x \in \partial V$ and $0<\lambda \leq 1$. Then, Fix $\mathfrak{F}$ is a nonempty compact set.

We state a generalization of Gronwall's lemma for singular kernels [22, Lemma 7.1.1]. Lemma 2.6. Let $v, w:[0, T] \rightarrow[0,+\infty)$ be continuous functions. If $w(\cdot)$ is nondecreasing and there are constants $a>0$ and $0<\alpha<1$ such that

$$
v(t) \leq w(t)+a \int_{0}^{t}(t-s)^{-\alpha} v(s) d s,
$$

then there exists a constant $k=k(\alpha)$ such that

$$
v(t) \leq w(t)+k a \int_{0}^{t}(t-s)^{-\alpha} w(s) d s, \quad \text { for each } t \in[0, T] .
$$


Based on the work in $[1,2,23]$, we set

$$
\begin{gathered}
Q(t)=\int_{0}^{\infty} \xi_{q}(\sigma) S\left(t^{q} \sigma\right) d \sigma, \\
R(t)=q \int_{0}^{\infty} \sigma t^{q-1} \xi_{q}(\sigma) S\left(t^{q} \sigma\right) d \sigma,
\end{gathered}
$$

and $\xi_{q}$ is a probability density function defined on $(0, \infty)$ such that

$$
\xi_{q}(\sigma)=\frac{1}{q} \sigma^{-1-(1 / q)} \varpi_{q}\left(\sigma^{-1 / q}\right) \geq 0
$$

where

$$
\varpi_{q}(\sigma)=\frac{1}{\pi} \sum_{n=1}^{\infty}(-1)^{n-1} \sigma^{-q n-1} \frac{\Gamma(n q+1)}{n !} \sin (n \pi q), \quad \sigma \in(0, \infty) .
$$

Remark 2.7 (see [23]). It is not difficult to verify that for $v \in[0,1]$,

$$
\int_{0}^{\infty} \sigma^{v} \xi_{q}(\sigma) d \sigma=\int_{0}^{\infty} \sigma^{-q v} \varpi_{q}(\sigma) d \sigma=\frac{\Gamma(1+v)}{\Gamma(1+q v)}
$$

Then, we can see

$$
\|R(t)\| \leq C_{q, M} t^{q-1}, \quad t>0
$$

where $C_{q, M}=q M / \Gamma(1+q)$.

We define the mild solution for problem (1.1) as follows.

Definition 2.8. A function $x \in C([-r, T], X)$ satisfying the equation

$$
x(t)= \begin{cases}g(x)(t)+\phi(t), & t \in[-r, 0], \\ Q(t)(g(x)(0)+\phi(0)-h(0, \phi+g(x)))+h\left(t, x_{t}\right) & \\ +\int_{0}^{t} \int_{0}^{s} R(t-s) K(s, \tau) f\left(\tau, x(\tau), x_{\tau}\right) d \tau d s, & t \in[0, T],\end{cases}
$$

is called a mild solution of problem (1.1). 


\section{Main Result}

We will require the following assumptions.

(H1) $f:[0, T] \times X \times C([-r, 0], X) \rightarrow X$ satisfies $f(\cdot, v, w):[0, T] \rightarrow X$ is measurable for all $(v, w) \in X \times C([-r, 0], X)$ and $f(t, \cdot, \cdot): X \times C([-r, 0], X) \rightarrow X$ is continuous for a.e. $t \in[0, T]$, and there exist two functions $\mu_{i}(\cdot) \in L^{1}\left([0, T], \mathbf{R}^{+}\right)(i=1,2)$ such that

$$
\|f(t, v, w)\| \leq \mu_{1}(t)\|v\|+\mu_{2}(t)\|w\|_{[-r, 0]}
$$

for almost all $t \in[0, T]$.

(H2) There exists a function $\eta \in L^{1}\left([0, T], \mathbf{R}^{+}\right)$such that for any bounded sets $D_{1} \subset X$, $D_{2} \subset C([-r, 0], X)$,

$$
\chi\left(f\left(\tau, D_{1}, D_{2}\right)\right) \leq \eta(\tau)\left(\chi\left(D_{1}\right)+\sup _{\theta \in[-r, 0]} \chi\left(D_{2}(\theta)\right)\right), \quad \text { a.e. } \tau \in[0, T] .
$$

(H3) (i) There exists a continuous function $L_{g}:[-r, 0] \rightarrow \mathbf{R}^{+}$such that

$$
\|g(x)(t)-g(y)(t)\| \leq L_{g}(t)\|x(t)-y(t)\|, \quad t \in[-r, 0]
$$

(ii) The function $g(x)(\cdot):[-r, 0] \rightarrow C([-r, 0], X)$ is equicontinuous and uniformly bounded, that is, there exists a constant $N>0$ such that

$$
\|g(x)\|_{[-r, 0]} \leq N \quad \forall x \in C([-r, 0], X) .
$$

(H4) (i) There exists a constant $L_{h}>0$ such that

$$
\left\|h\left(t_{1}, \varphi\right)-h\left(t_{2}, \tilde{\varphi}\right)\right\| \leq L_{h}\left(\left|t_{1}-t_{2}\right|+\|\varphi-\tilde{\varphi}\|_{[-r, 0]}\right), \quad t_{1}, t_{2} \in[0, T], \varphi, \tilde{\varphi} \in C([-r, 0], X) .
$$

(ii) For every bounded set $\Omega \subset C([-r, 0], X)$ and $t \in[0, T]$, there exists a constant $0<\omega<1$ such that

$$
x(h(t, \Omega)) \leq \omega \sup _{s \in[-r, 0]} x(\Omega(s)) .
$$

(H5) For each $t \in[0, T], K(t, \cdot)$ is measurable on $[0, t]$ and $K(t)=\operatorname{ess} \sup \{|K(t, s)|, 0 \leq$ $s \leq t\}$ is bounded on $[0, T]$. The map $t \rightarrow K_{t}$ is continuous from $[0, T]$ to $L^{\infty}([0, T], \mathbf{R})$, here, $K_{t}(s)=K(t, s)$.

(H6) There exists $M^{*} \in(0,1)$ such that

$$
L_{0}+\frac{M K T^{q}}{\Gamma(q+1)} \max \left\{2\|\eta\|_{L^{1}}\left\|\mu_{1}\right\|_{L^{1}}+\left\|\mu_{2}\right\|_{L^{1}}\right\}<M^{*}
$$

where $L_{0}=M L_{g}^{*}(1+\omega)+\max \left\{L_{h}, \omega\right\}, L_{g}^{*}=\sup _{t \in[-r, 0]} L_{g}(t), K=\sup _{t \in[0, T]} K(t)$. 
Theorem 3.1. Assume that (H1)-(H6) are satisfied. Then, the mild solutions set of problem (1.1) is a nonempty compact subset of the space $C([-r, T], X)$.

Proof. Define the operator $\Lambda: C([-r, T], X) \rightarrow C([-r, T], X)$ in the following way:

$$
(\Lambda x)(t)= \begin{cases}g(x)(t)+\phi(t), & t \in[-r, 0], \\ Q(t)(g(x)(0)+\phi(0)-h(0, \phi+g(x)))+h\left(t, x_{t}\right) & \\ +\int_{0}^{t} \int_{0}^{s} R(t-s) K(s, \tau) f\left(\tau, x(\tau), x_{\tau}\right) d \tau d s, & t \in[0, T] .\end{cases}
$$

It is clear that the operator $\Lambda$ is well defined, and the fixed point of $\Lambda$ is the mild solution of problem (1.1).

The operator $\Lambda$ can be written in the form $\Lambda=\sum_{i=1}^{3} \Lambda_{i}$, where the operators $\Lambda_{i}, i=$ $1,2,3$ are defined as follows:

$$
\begin{aligned}
& \left(\Lambda_{1} x\right)(t)= \begin{cases}g(x)(t)+\phi(t), & t \in[-r, 0], \\
Q(t)(g(x)(0)+\phi(0)), & t \in[0, T],\end{cases} \\
& \left(\Lambda_{2} x\right)(t)= \begin{cases}0, & t \in[-r, 0], \\
-Q(t) h(0, \phi+g(x))+h\left(t, x_{t}\right), & t \in[0, T],\end{cases} \\
& \left(\Lambda_{3} x\right)(t)= \begin{cases}0, & t \in[-r, 0], \\
\int_{0}^{t} \int_{0}^{s} R(t-s) K(s, \tau) f\left(\tau, x(\tau), x_{\tau}\right) d \tau d s, & t \in[0, T] .\end{cases}
\end{aligned}
$$

Obviously, under the assumptions of $g$ and $h, \Lambda_{1}$ and $\Lambda_{2}$ are continuous, respectively. For $t \in[0, T]$, we can prove that $\Lambda_{3}$ is continuous.

Indeed, let $\left\{x^{k}\right\}_{k \in \mathbf{N}}$ be a sequence such that $x^{k} \rightarrow x$ in $C([-r, T], X)$ as $k \rightarrow \infty$. Since $f$ satisfies (H1), for almost every $t \in[0, T]$, we get

$$
f\left(t, x^{k}(t), x_{t}^{k}\right) \longrightarrow f\left(t, x(t), x_{t}\right), \quad \text { as } k \longrightarrow \infty
$$

Noting that $x^{k} \rightarrow x$ in $C([-r, T], X)$, we can see that there exists $\varepsilon>0$ such that $\left\|x^{k}-x\right\|_{[-r, T]} \leq \varepsilon$ for $k$ sufficiently large. Therefore, we have

$$
\begin{aligned}
\| f(t, & \left.x^{k}(t), x_{t}^{k}\right)-f\left(t, x(t), x_{t}\right) \| \\
\leq & \mu_{1}(t)\left\|x^{k}(t)\right\|+\mu_{2}(t)\left\|x_{t}^{k}\right\|_{[-r, 0]}+\mu_{1}(t)\|x(t)\|+\mu_{2}(t)\left\|x_{t}\right\|_{[-r, 0]} \\
\leq & \mu_{1}(t)\left\|x^{k}(t)-x(t)\right\|+\mu_{1}(t)\|x(t)\|+\mu_{2}(t)\left\|x_{t}^{k}-x_{t}\right\|_{[-r, 0]}+\mu_{2}(t)\left\|x_{t}\right\|_{[-r, 0]} \\
& \quad+\mu_{1}(t)\|x(t)\|+\mu_{2}(t)\left\|x_{t}\right\|_{[-r, 0]} \\
= & \mu_{1}(t)\left\|x^{k}(t)-x(t)\right\|+2 \mu_{1}(t)\|x(t)\|+\mu_{2}(t)\left\|x_{t}^{k}-x_{t}\right\|_{[-r, 0]}+2 \mu_{2}(t)\left\|x_{t}\right\|_{[-r, 0]} \\
\leq & \mu_{1}(t) \varepsilon+2 \mu_{1}(t)\|x\|_{[0, T]}+\mu_{2}(t) \varepsilon+2 \mu_{2}(t)\|x\|_{[-r, T]} \\
\leq & \left(\mu_{1}(t)+\mu_{2}(t)\right) \varepsilon+2\left(\mu_{1}(t)+\mu_{2}(t)\right)\|x\|_{[-r, T]} .
\end{aligned}
$$


It follows from the Lebesgue's Dominated Convergence Theorem that

$$
\begin{aligned}
& \left\|\left(\Lambda_{3} x^{k}\right)(t)-\left(\Lambda_{3} x\right)(t)\right\| \\
& \quad \leq \int_{0}^{t} \int_{0}^{s}\left\|R(t-s) K(s, \tau)\left[f\left(\tau, x^{k}(\tau), x_{\tau}^{k}\right)-f\left(\tau, x(\tau), x_{\tau}\right)\right]\right\| d \tau d s \\
& \quad \leq K C_{q, M} \int_{0}^{t} \int_{0}^{s}(t-s)^{q-1}\left\|f\left(\tau, x^{k}(\tau), x_{\tau}^{k}\right)-f\left(\tau, x(\tau), x_{\tau}\right)\right\| d \tau d s \\
& \quad \rightarrow 0, \quad \text { as } k \longrightarrow \infty .
\end{aligned}
$$

Therefore, we obtain that

$$
\lim _{k \rightarrow \infty}\left\|\Lambda_{3} x^{k}-\Lambda_{3} x\right\|_{[-r, T]}=0
$$

This shows that $\Lambda_{3}$ is continuous. Therefore, $\Lambda$ is continuous.

Consider the set

$$
B_{\rho}=\left\{x \in C([-r, T], X):\|x\|_{[-r, T]} \leq \rho\right\}
$$

In view of (H4), for $x \in B_{\rho}$,

$$
\begin{aligned}
\left\|h\left(t, x_{t}\right)\right\| & \leq\left\|h\left(t, x_{t}\right)-h(t, 0)\right\|+\|h(t, 0)\| \\
& \leq L_{h} \rho+M_{1}
\end{aligned}
$$

where $M_{1}=\sup _{t \in[0, T]}\|h(t, 0)\|$.

Next, we show that there exists some $\rho>0$ such that $\Lambda B_{\rho} \subset B_{\rho}$. Suppose, on the contrary, that for each $\rho>0$, there exist $x^{\rho}(\cdot) \in B_{\rho}$ and some $t \in[-r, T]$ such that $\left\|\left(\Lambda x^{\rho}\right)(t)\right\|>$ $\rho$. Now, if $t \in[-r, 0]$, then

$$
\begin{aligned}
\rho & <\left\|\left(\Lambda x^{\rho}\right)(t)\right\|=\left\|g\left(x^{\rho}\right)(t)+\phi(t)\right\| \\
& \leq N+\|\phi\|_{[-r, 0]}
\end{aligned}
$$

and if $t \in[0, T]$, then

$$
\begin{aligned}
\rho< & \left\|\left(\Lambda x^{\rho}\right)(t)\right\| \leq\left\|\left(\Lambda_{1} x^{\rho}\right)(t)\right\|+\left\|\left(\Lambda_{2} x^{\rho}\right)(t)\right\|+\left\|\left(\Lambda_{3} x^{\rho}\right)(t)\right\| \\
\leq & \left\|Q(t)\left(g\left(x^{\rho}\right)(0)+\phi(0)\right)\right\|+\left\|-Q(t) h\left(0, \phi+g\left(x^{\rho}\right)\right)+h\left(t, x_{t}^{\rho}\right)\right\| \\
& +\int_{0}^{t} \int_{0}^{s}\left\|R(t-s) K(s, \tau) f\left(\tau, x^{\rho}(\tau), x_{\tau}^{\rho}\right)\right\| d \tau d s \\
\leq & M(N+\|\phi(0)\|)+M L_{h}\left(\|\phi\|_{[-r, 0]}+N\right)+\rho L_{h}+M_{1}(M+1) \\
& +\rho C_{q, M} K \int_{0}^{t}(t-s)^{q-1} \int_{0}^{s}\left(\mu_{1}(\tau)+\mu_{2}(\tau)\right) d \tau d s \\
\leq & M_{2}+\rho L_{h}+\frac{T^{q} K M \rho}{\Gamma(q+1)}\left(\left\|\mu_{1}\right\|_{L^{1}}+\left\|\mu_{2}\right\|_{L^{1}}\right),
\end{aligned}
$$

where $M_{2}:=M(N+\|\phi(0)\|)+M L_{h}\left(\|\phi\|_{[-r, 0]}+N\right)+M_{1}(M+1)$. 
Denote by $L_{\rho}$ the right-hand side of (3.17), then we have

$$
\rho \leq \max \left\{\|\phi\|_{[-r, 0]}+N, L_{\rho}\right\} .
$$

Dividing both sides of (3.18) by $\rho$ and taking $\rho \rightarrow \infty$, we have

$$
L_{h}+\frac{T^{q} K M}{\Gamma(q+1)}\left(\left\|\mu_{1}\right\|_{L^{1}}+\left\|\mu_{2}\right\|_{L^{1}}\right) \geq 1
$$

This contradicts (H6). Hence, for some positive number, $\rho, \Lambda B_{\rho} \subset B_{\rho}$.

Let $\chi$ be a Hausdorff MNC in $X$; we consider the measure of noncompactness $\beta$ in the space $C([-r, T], X)$ with values in the cone $\mathbf{R}_{+}^{2}$ of the following way: for every bounded subset $\Omega \subset C([-r, T], X)$,

$$
\beta(\Omega)=\left(\Psi(\Omega), \bmod _{c}(\Omega)\right)
$$

where

$$
\Psi(\Omega)=\sup _{t \in[-r, T]} X(\Omega(t))
$$

and $\bmod _{c}(\Omega)$ is the module of equicontinuity of $\Omega$ given by

$$
\bmod _{c}(\Omega)=\lim _{\delta \rightarrow 0} \sup _{x \in \Omega} \max _{\left|t_{1}-t_{2}\right| \leq \delta}\left\|x\left(t_{1}\right)-x\left(t_{2}\right)\right\|
$$

Next, we show that the operator $\Lambda$ is $\beta$-condensing on every bounded subset of $C([-r, T], X)$.

Let $\Omega \subset C([-r, T], X)$ be a nonempty, bounded set such that

$$
\beta(\Lambda(\Omega)) \geq \beta(\Omega)
$$

Firstly, we estimate $\Psi(\Omega)$. For $t \in[-r, 0], x, y \in \Omega$, we have

$$
\|g(x)(t)-g(y)(t)\| \leq L_{g}^{*}\|x(t)-y(t)\|,
$$

if we denote $\chi(\Omega[a, b]):=\sup _{t \in[a, b]} \mathcal{X}(\Omega(t))$, then

$$
\chi(g(\Omega)[-r, 0]) \leq L_{g}^{*} X(\Omega[-r, 0]) .
$$

Then,

$$
\chi((\Lambda \Omega)([-r, 0]))=\chi(\{\phi([-r, 0])\}+g(\Omega)[-r, 0])=\chi(g(\Omega)[-r, 0]) \leq L_{g}^{*} \chi(\Omega[-r, 0]),
$$


by (3.23), we can see that

$$
\chi(\Omega[-r, 0])=0 .
$$

Furthermore, $\chi((\Lambda \Omega)([-r, 0]))=0$.

For $t \in[0, T]$, one gets

$$
\left\|\left(\Lambda_{1} x\right)(t)-\left(\Lambda_{1} y\right)(t)\right\| \leq\|Q(t)\|\|g(x)(0)-g(y)(0)\| \leq M L_{g}^{*}\|x(0)-y(0)\|,
$$

thus $X\left(\left(\Lambda_{1} \Omega\right)([0, T])\right) \leq M L_{g}^{*} \Psi(\Omega)$.

Moreover, we see that

$$
\begin{gathered}
\chi(-Q(t) h(0, \phi+g(\Omega))) \leq M \omega \sup _{s \in[-r, 0]} \chi(g(\Omega)(s)) \leq M \omega L_{g}^{*} \Psi(\Omega), \\
\chi\left(h\left(t, \Omega_{t}\right)\right) \leq \omega \sup _{-r \leq \theta \leq 0} \chi(\Omega(t+\theta)) \leq \omega \Psi(\Omega),
\end{gathered}
$$

where $\Omega_{t}=\left\{x_{t}: x \in \Omega\right\}$. Now, we can see $\chi\left(\left(\Lambda_{2} \Omega\right)([0, T])\right) \leq \omega\left(M L_{g}^{*}+1\right) \Psi(\Omega)$.

For any $t \in[0, T]$, we set

$$
\Phi(\Omega)(t)=\left\{\int_{0}^{t} \int_{0}^{s} R(t-s) K(s, \tau) f\left(\tau, x(\tau), x_{\tau}\right) d \tau d s: x \in \Omega\right\} .
$$

We consider the multifunction $s \in[0, t] \multimap G(s)$,

$$
G(s)=\left\{R(t-s) \int_{0}^{s} K(s, \tau) f\left(\tau, x(\tau), x_{\tau}\right) d \tau: x \in \Omega\right\}
$$

Obviously, G is integrable, and, from (H1), it follows that it is integrably bounded. Moreover, noting that $(\mathrm{H} 2)$ and Proposition 2.2, we have the following estimate for a.e. $s \in[0, t]$ :

$$
\begin{aligned}
x(G(s)) & \leq C_{q, M}(t-s)^{q-1} K \cdot x\left(\left\{\int_{0}^{s} f\left(\tau, x(\tau), x_{\tau}\right) d \tau: x \in \Omega\right\}\right) \\
& =C_{q, M}(t-s)^{q-1} K \cdot x\left(\int_{0}^{s} f\left(\tau, \Omega(\tau), \Omega_{\tau}\right) d \tau\right) \\
& \leq C_{q, M}(t-s)^{q-1} K \cdot \int_{0}^{s}\left[\eta(\tau)\left(x(\Omega(\tau))+\sup _{\theta \in[-r, 0]} x(\Omega(\tau+\theta))\right)\right] d \tau .
\end{aligned}
$$

Moreover, the equality (3.27) implies that

$$
\sup _{-r \leq \sigma \leq 0} X(\Omega(\sigma))=0
$$


Therefore, for $\tau \in[0, T]$, we have

$$
\sup _{\tau-r \leq \sigma \leq \tau} \chi(\Omega(\sigma)) \leq \sup _{-r \leq \sigma \leq 0} \chi(\Omega(\sigma))+\sup _{0 \leq \sigma \leq \tau} \chi(\Omega(\sigma))=\sup _{0 \leq \sigma \leq \tau} \chi(\Omega(\sigma))
$$

then we can see

$$
\chi(G(s)) \leq 2 C_{q, M}(t-s)^{q-1} K \cdot\|\eta\|_{L^{1}} \sup _{0 \leq \theta \leq t} X(\Omega(\theta)) \leq 2 C_{q, M}(t-s)^{q-1} K \cdot\|\eta\|_{L^{1}} \cdot \Psi(\Omega) .
$$

Applying Proposition 2.2, we have

$$
\chi(\Phi(\Omega)(t))=\chi\left(\int_{0}^{t} G(s) d s\right) \leq \frac{2 M K T^{q}}{\Gamma(q+1)} \cdot\|\eta\|_{L^{1}} \cdot \Psi(\Omega)
$$

that is,

$$
\chi\left(\left(\Lambda_{3} \Omega\right)([0, T])\right) \leq \frac{2 M K T^{q}}{\Gamma(q+1)}\|\eta\|_{L^{1}} \Psi(\Omega)
$$

Now, we can see

$$
\begin{aligned}
\chi((\Lambda \Omega)([0, T])) & \leq X\left(\left(\Lambda_{1} \Omega\right)([0, T])\right)+\chi\left(\left(\Lambda_{2} \Omega\right)([0, T])\right)+\chi\left(\left(\Lambda_{3} \Omega\right)([0, T])\right) \\
& \leq\left(M L_{g}^{*}(1+\omega)+\omega+\frac{2 M K T^{q}}{\Gamma(q+1)}\|\eta\|_{L^{1}}\right) \Psi(\Omega),
\end{aligned}
$$

furthermore

$$
\Psi(\Lambda \Omega) \leq\left(M L_{g}^{*}(1+\omega)+\omega+\frac{2 M K T^{q}}{\Gamma(q+1)}\|\eta\|_{L^{1}}\right) \Psi(\Omega)
$$

which implies, by (H6) and (3.23), $\Psi(\Omega)=0$. Next, we will prove $\bmod _{c}(\Omega)=0$.

Noting (H3) (ii) and the continuity of $\{S(t)\}_{t \geq 0}$ in the uniform operator topology for $t>0$, we can see

$$
\bmod _{c}\left(\Lambda_{1} \Omega\right)=0 .
$$


Let $\delta>0, t_{1}, t_{2} \in[0, T]$ such that $0<\left|t_{1}-t_{2}\right| \leq \delta$ and $x \in \Omega$, we obtain

$$
\begin{aligned}
\left\|h\left(t_{1}, x_{t_{1}}\right)-h\left(t_{2}, x_{t_{2}}\right)\right\| & \leq L_{h}\left(\left|t_{1}-t_{2}\right|+\left\|x_{t_{1}}-x_{t_{2}}\right\|_{[-r, 0]}\right) \\
& =L_{h}\left(\left|t_{1}-t_{2}\right|+\sup _{\theta \in[-r, 0],\left|t_{1}-t_{2}\right| \leq \delta}\left\|x\left(t_{1}+\theta\right)-x\left(t_{2}+\theta\right)\right\|\right) \\
& \leq L_{h}\left(\left|t_{1}-t_{2}\right|+\sup _{s_{1}, s_{2} \in[-r, T],\left|s_{1}-s_{2}\right| \leq \delta}\left\|x\left(s_{1}\right)-x\left(s_{2}\right)\right\|\right) .
\end{aligned}
$$

Moreover, noting the continuity of $\{S(t)\}_{t \geq 0}$ in the uniform operator topology for $t>0$, then we have

$$
\bmod _{c}\left(\Lambda_{2} \Omega\right) \leq L_{h} \bmod _{c}(\Omega)
$$

For $0<t_{2}<t_{1}<T$ and $x \in \Omega$, we have

$$
\left\|\left(\Lambda_{3} x\right)\left(t_{1}\right)-\left(\Lambda_{3} x\right)\left(t_{2}\right)\right\| \leq I_{1}+I_{2}
$$

where

$$
\begin{gathered}
I_{1}=K \int_{0}^{t_{2}} \int_{0}^{s}\left\|\left[R\left(t_{1}-s\right)-R\left(t_{2}-s\right)\right] f\left(\tau, x(\tau), x_{\tau}\right)\right\| d \tau d s, \\
I_{2}=K \int_{t_{2}}^{t_{1}} \int_{0}^{s}\left\|R\left(t_{1}-s\right)\right\|\left\|f\left(\tau, x(\tau), x_{\tau}\right)\right\| d \tau d s .
\end{gathered}
$$

For $I_{1}$, we have

$$
\begin{aligned}
& I_{1} \leq q K \int_{0}^{t_{2}} \int_{0}^{\infty} \sigma\left\|\left[\left(t_{1}-s\right)^{q-1}-\left(t_{2}-s\right)^{q-1}\right] \xi_{q}(\sigma) S\left(\left(t_{1}-s\right)^{q} \sigma\right) \int_{0}^{s} f\left(\tau, x(\tau), x_{\tau}\right) d \tau\right\| d \sigma d s \\
& \quad+q K \int_{0}^{t_{2}} \int_{0}^{\infty} \sigma\left(t_{2}-s\right)^{q-1} \xi_{q}(\sigma)\left\|S\left(\left(t_{1}-s\right)^{q} \sigma\right)-S\left(\left(t_{2}-s\right)^{q} \sigma\right)\right\| \int_{0}^{s}\left\|f\left(\tau, x(\tau), x_{\tau}\right)\right\| d \tau d \sigma d s \\
& \leq C_{q, M} K \int_{0}^{t_{2}}\left|\left(t_{1}-s\right)^{q-1}-\left(t_{2}-s\right)^{q-1}\right| \int_{0}^{s}\left(\mu_{1}(\tau)\|x(\tau)\|+\mu_{2}(\tau)\left\|x_{\tau}\right\|_{[-r, 0]}\right) d \tau d s \\
& \quad+q K \int_{0}^{t_{2}} \int_{0}^{\infty} \sigma\left(t_{2}-s\right)^{q-1} \xi_{q}(\sigma)\left\|S\left(\left(t_{1}-s\right)^{q} \sigma\right)-S\left(\left(t_{2}-s\right)^{q} \sigma\right)\right\| \int_{0}^{s}\left\|f\left(\tau, x(\tau), x_{\tau}\right)\right\| d \tau d \sigma d s \\
& \leq K\left(\left\|\mu_{1}\right\|_{L^{1}}+\left\|\mu_{2}\right\|_{L^{1}}\right)\|x\|_{[-r, T]} \cdot\left[C_{q, M} \int_{0}^{t_{2}}\left|\left(t_{1}-s\right)^{q-1}-\left(t_{2}-s\right)^{q-1}\right| d s+q \int_{0}^{t_{2}} \int_{0}^{\infty} \sigma\left(t_{2}-s\right)^{q-1}\right. \\
& \left.\times \xi_{q}(\sigma)\left\|S\left(\left(t_{1}-s\right)^{q} \sigma\right)-S\left(\left(t_{2}-s\right)^{q} \sigma\right)\right\| d \sigma d s\right] .
\end{aligned}
$$


Clearly, the first term on the right-hand side of (3.45) tends to 0 as $t_{2} \rightarrow t_{1}$. The second term on the right-hand side of (3.45) tends to 0 as $t_{2} \rightarrow t_{1}$ as a consequence of the continuity of $S(t)$ in the uniform operator topology for $t>0$.

In view of the assumption of $\mu_{i}(s)(i=1,2)$, we see that

$$
\begin{aligned}
I_{2} & =K \int_{t_{2}}^{t_{1}} \int_{0}^{s}\left\|R\left(t_{1}-s\right)\right\|\left\|f\left(\tau, x(\tau), x_{\tau}\right)\right\| d \tau d s \\
& \leq K C_{q, M}\left(\left\|\mu_{1}\right\|_{L^{1}}+\left\|\mu_{2}\right\|_{L^{1}}\right)\|x\|_{[-r, T]} \int_{t_{2}}^{t_{1}}\left(t_{1}-s\right)^{q-1} d s \\
& \rightarrow 0, \quad \text { as } t_{2} \longrightarrow t_{1} .
\end{aligned}
$$

Thus, the set $\left\{\left(\Lambda_{3} x\right)(\cdot): x \in \Omega\right\}$ is equicontinuous, then $\bmod _{c}\left(\Lambda_{3} \Omega\right)=0$.

Since

$$
\bmod _{c}(\Lambda \Omega) \leq \sum_{i=1}^{3} \bmod _{c}\left(\Lambda_{i} \Omega\right) \leq L_{0} \bmod _{c}(\Omega),
$$

then $\bmod _{c}(\Omega)=0$, which yields from (3.23), hence

$$
\beta(\Omega)=(0,0)
$$

The regularity property of $\beta$ implies the relative compactness of $\Omega$.

Now, it follows from Definition 2.3 that $\Lambda$ is $\beta$-condensing.

According to Theorem 2.4, problem (1.1) has at least one mild solution.

Next, for $\delta \in(0,1]$, we consider the following one-parameter family of maps:

$$
\begin{gathered}
\Pi:[0,1] \times C([-r, T], X) \longrightarrow C([-r, T], X), \\
(\delta, x) \longrightarrow \Pi(\delta, x)=\delta \Lambda(x) .
\end{gathered}
$$

We will prove that the fixed point set of the family $\Pi$,

$$
\text { Fix } \Pi=\{x \in \Pi(\delta, x) \text { for some } \delta \in(0,1]\}
$$

is a priori bounded.

Let $x \in$ Fix $\Pi$, for $t \in[0, T]$, we have

$$
\begin{aligned}
\left\|x_{t}\right\|_{[-r, 0]} & =\sup _{-r \leq \theta \leq 0}\|x(t+\theta)\| \leq \sup _{-r \leq \tau \leq 0}\|x(\tau)\|+\sup _{0 \leq \tau \leq t}\|x(\tau)\| \\
& \leq N+\|\phi\|_{[-r, 0]}+\sup _{0 \leq \tau \leq t}\|x(\tau)\| .
\end{aligned}
$$


Then,

$$
\begin{aligned}
\|x(t)\| \leq & \|Q(t)(g(x)(0)+\phi(0))\|+\|-Q(t) h(0, \phi+g(x))\|+\left\|h\left(t, x_{t}\right)\right\| \\
& +\int_{0}^{t} \int_{0}^{s}\left\|R(t-s) K(s, \tau) f\left(\tau, x(\tau), x_{\tau}\right)\right\| d \tau d s \\
\leq & M(N+\|\phi(0)\|)+L_{h}(M+1)\left(\|\phi\|_{[-r, 0]}+N\right)+M_{1}(M+1)+L_{h} \sup _{0 \leq \tau \leq t}\|x(\tau)\| \\
& +C_{q, M} K \int_{0}^{t}(t-s)^{q-1} \int_{0}^{s}\left[\mu_{1}(\tau)\|x(\tau)\|+\mu_{2}(\tau)\left(\|\phi\|_{[-r, 0]}+N+\sup _{0 \leq \theta \leq \tau}\|x(\theta)\|\right)\right] d \tau d s \\
\leq & \theta_{1}+L_{h} \sup _{0 \leq \tau \leq t}\|x(\tau)\|+C_{q, M} K\left(\left\|\mu_{1}\right\|_{L^{1}}+\left\|\mu_{2}\right\|_{L^{1}}\right) \int_{0}^{t}(t-s)^{q-1} \sup _{0 \leq \tau \leq s}\|x(\tau)\| d s \\
= & \theta_{1}+L_{h} \sup _{0 \leq \tau \leq t}\|x(\tau)\|+\theta_{2} \int_{0}^{t}(t-s)^{q-1} \sup _{0 \leq \tau \leq s}\|x(\tau)\| d s,
\end{aligned}
$$

where

$$
\begin{aligned}
\theta_{1} & :=L+\frac{M K T^{q}\left\|\mu_{2}\right\|_{L^{1}}}{\Gamma(q+1)}\left(\|\phi\|_{[-r, 0]}+N\right) \\
L & :=M(N+\|\phi(0)\|)+L_{h}(M+1)\left(\|\phi\|_{[-r, 0]}+N\right)+M_{1}(M+1), \\
\theta_{2} & :=C_{q, M} K\left(\left\|\mu_{1}\right\|_{L^{1}}+\left\|\mu_{2}\right\|_{L^{1}}\right) .
\end{aligned}
$$

We denote $\kappa(t):=\sup _{0 \leq s \leq t}\|x(s)\|$. Let $\tilde{t} \in[0, t]$ such that $\kappa(t)=\|x(\tilde{t})\|$. Then, by (3.52), we can see

$$
\kappa(t) \leq \theta_{1}+L_{h} \mathcal{\kappa}(t)+\theta_{2} \int_{0}^{t}(t-s)^{q-1} \kappa(s) d s .
$$

By Lemma 2.6, there exists a constant $\tilde{k}=\tilde{k}(q)$ such that

$$
\kappa(t) \leq \frac{\theta_{1}}{1-L_{h}}+\frac{\tilde{k} \theta_{1} \theta_{2}}{\left(1-L_{h}\right)^{2}} \int_{0}^{t}(t-s)^{q-1} d s \leq \frac{\theta_{1}}{1-L_{h}}+\frac{\tilde{k} \theta_{1} \theta_{2} T^{q}}{q\left(1-L_{h}\right)^{2}}:=\zeta .
$$

Therefore,

$$
\begin{aligned}
\sup _{t \in[-r, T]}\|x(t)\| & \leq \sup _{t \in[-r, 0]}\|x(t)\|+\sup _{t \in[0, T]}\|x(t)\| \\
& =\sup _{t \in[-r, 0]}\|g(x)(t)+\phi(t)\|+\sup _{t \in[0, T]}\|x(t)\| \\
& \leq N+\|\phi\|_{[-r, 0]}+\zeta .
\end{aligned}
$$


Now, we consider a closed ball

$$
B_{R}=\left\{x \in C([-r, T], X):\|x\|_{[-r, T]} \leq R\right\} \subset C([-r, T], X) .
$$

We take the radius $R>0$ large enough to contain the set Fix $\Pi$ inside itself. Moreover, from the proof above, $\Lambda: B_{R} \rightarrow C([-r, T], X)$ is $\beta$-condensing and it remains to apply Theorem 2.5.

\section{Applications}

Example 4.1. In this section, we consider the following integrodifferential model:

$$
\begin{gathered}
\frac{\partial^{q}}{\partial t^{q}}\left[v(t, \xi)-e^{-t} \int_{-r}^{0} \frac{\gamma_{1}(\theta)}{1+|v(t+\theta, \xi)|} d \theta\right] \\
=\frac{\partial^{2}}{\partial \xi^{2}}\left[v(t, \xi)-e^{-t} \int_{-r}^{0} \frac{\gamma_{1}(\theta)}{1+|v(t+\theta, \xi)|} d \theta\right] \\
+\int_{0}^{t}(t-s) s^{k} \sin |v(s, \xi)| d s+\int_{0}^{t}(t-s) \int_{-r}^{0} \gamma_{2}(\theta) s^{2 / 3} \cdot \sin \left(\frac{|v(s+\theta, \xi)|}{s}\right) d \theta d s, \\
v(t, 0)-e^{-t} \int_{-r}^{0} \frac{\gamma_{1}(\theta)}{1+|v(t+\theta, 0)|} d \theta=0, \\
v(t, 1)-e^{-t} \int_{-r}^{0} \frac{\gamma_{1}(\theta)}{1+|v(t+\theta, 1)|} d \theta=0, \\
v(\theta, \xi)=v_{0}(\theta, \xi)+\frac{e^{\mu \theta}}{k^{2}} \cdot \frac{|v(\theta, \xi)|}{1+|v(\theta, \xi)|}, \quad-r \leq \theta \leq 0,
\end{gathered}
$$

where $0 \leq t \leq 1, \xi \in[0,1], k \in \mathbf{N}, r>0, \mu>0, \gamma_{1}:[-r, 0] \rightarrow \mathbf{R}, \gamma_{2}:[-r, 0] \rightarrow \mathbf{R}$, $v_{0}:[-r, 0] \times[0,1] \rightarrow \mathbf{R}$ are continuous functions, and $\int_{-r}^{0}\left|\gamma_{1}(\theta)\right| d \theta<1, \int_{-r}^{0}\left|\gamma_{2}(\theta)\right| d \theta<\infty$.

Set $X=L^{2}([0,1], \mathbf{R})$ and define $A$ by

$$
\begin{gathered}
D(A)=H^{2}(0,1) \cap H_{0}^{1}(0,1), \\
A u=u^{\prime \prime} .
\end{gathered}
$$

Then, $A$ generates a compact, analytic semigroup $S(\cdot)$ of uniformly bounded linear operators, and $\|S(t)\| \leq 1$.

For $\xi \in[0,1]$ and $\varphi \in C([-r, 0], X)$, we set

$$
\begin{gathered}
x(t)(\xi)=v(t, \xi), \\
\phi(\theta)(\xi)=v_{0}(\theta, \xi), \quad \theta \in[-r, 0], \\
h(t, \varphi)(\xi)=e^{-t} \int_{-r}^{0} \frac{\gamma_{1}(\theta)}{1+|\varphi(\theta)(\xi)|} d \theta, \\
g(\varphi(\theta))(\xi)=\frac{e^{\mu \theta}}{k^{2}} \cdot \frac{|\varphi(\theta)(\xi)|}{1+|\varphi(\theta)(\xi)|} \\
K(t, s)=t-s, \\
f(t, x(t), \varphi)(\xi)=t^{k} \sin |x(t)(\xi)|+\int_{-r}^{0} r_{2}(\theta) t^{2 / 3} \cdot \sin \left(\frac{|\varphi(\theta)(\xi)|}{t}\right) d \theta .
\end{gathered}
$$

Then, (4.1) can be reformulated as the abstract (1.1). 
Moreover, for $t \in(0,1]$, we can see

$$
\begin{aligned}
\|f(t, x(t), \varphi)\| & \leq t^{k}\|x(t)\|+\frac{1}{\sqrt[3]{t}}\|\varphi\|_{[-r, 0]} \int_{-r}^{0}\left|\gamma_{2}(\theta)\right| d \theta \\
& =\mu_{1}(t)\|x(t)\|+\mu_{2}(t)\|\varphi\|_{[-r, 0]^{\prime}}
\end{aligned}
$$

where $\mu_{1}(t):=t^{k}, \mu_{2}(t):=(1 / \sqrt[3]{t}) \int_{-r}^{0}\left|\gamma_{2}(\theta)\right| d \theta$.

For any $x_{1}, x_{2} \in X, \varphi, \tilde{\varphi} \in C([-r, 0], X)$,

$$
\begin{aligned}
& \left\|f\left(t, x_{1}(t), \varphi\right)(\xi)-f\left(t, x_{2}(t), \tilde{\varphi}\right)(\xi)\right\| \\
& \quad \leq t^{k}\left\|x_{1}(t)-x_{2}(t)\right\|+\frac{1}{\sqrt[3]{t}} \int_{-r}^{0}\left|\gamma_{2}(\theta)\right|\|\varphi(\theta)(\xi)-\tilde{\varphi}(\theta)(\xi)\| d \theta .
\end{aligned}
$$

Therefore, for any bounded sets $D_{1} \subset X, D_{2} \subset C([-r, 0], X)$, we have

$$
\begin{aligned}
x\left(f\left(t, D_{1}, D_{2}\right)\right) & \leq t^{k} \cdot x\left(D_{1}\right)+\frac{1}{\sqrt[3]{t}} \int_{-r}^{0}\left|\gamma_{2}(\theta)\right| x\left(D_{2}(\theta)\right) d \theta \\
& \leq t^{k} \cdot x\left(D_{1}\right)+\frac{1}{\sqrt[3]{t}} \sup _{-r \leq \theta \leq 0} x\left(D_{2}(\theta)\right) \int_{-r}^{0}\left|\gamma_{2}(\theta)\right| d \theta \\
& \leq \eta(t)\left(x\left(D_{1}\right)+\sup _{-r \leq \theta \leq 0} x\left(D_{2}(\theta)\right)\right), \quad \text { a.e. } t \in[0,1]
\end{aligned}
$$

where $\eta(t):=\max \left\{t^{k},(1 / \sqrt[3]{t}) \int_{-r}^{0}\left|\gamma_{2}(\theta)\right| d \theta\right\}$.

For $\varphi, \tilde{\varphi} \in C([-r, 0], X), \theta \in[-r, 0]$, we can see

$$
\|g(\varphi)(\xi)-g(\widetilde{\varphi})(\xi)\| \leq \frac{e^{\mu \theta}}{k^{2}} \cdot\|\varphi-\tilde{\varphi}\| \leq \frac{1}{k^{2}} \cdot\|\varphi-\tilde{\varphi}\| .
$$

We denote $L_{g}:=1 / k^{2}$. Moreover,

$$
\|g(\varphi)(\xi)\| \leq \frac{1}{k^{2}}:=N .
$$

For $t_{1}, t_{2} \in[0,1], \varphi, \tilde{\varphi} \in C([-r, 0], X)$, we have

$$
\begin{aligned}
& \left\|h\left(t_{1}, \varphi\right)-h\left(t_{2}, \tilde{\varphi}\right)\right\| \\
& \leq\left|t_{1}-t_{2}\right| \int_{-r}^{0}\left\|\frac{\gamma_{1}(\theta)}{1+|\varphi(\theta)(\xi)|}\right\| d \theta \\
& \quad+e^{-t_{2}} \int_{-r}^{0}\left\|\gamma_{1}(\theta)\left(\frac{1}{1+|\varphi(\theta)(\xi)|}-\frac{1}{1+|\tilde{\varphi}(\theta)(\xi)|}\right)\right\| d \theta \\
& \leq\left|t_{1}-t_{2}\right| \int_{-r}^{0}\left|\gamma_{1}(\theta)\right| d \theta+\int_{-r}^{0}\left|\gamma_{1}(\theta)\right|\|\varphi(\theta)(\xi)-\tilde{\varphi}(\theta)(\xi)\| d \theta \\
& \leq\left|t_{1}-t_{2}\right| \int_{-r}^{0}\left|\gamma_{1}(\theta)\right| d \theta+\int_{-r}^{0}\left|\gamma_{1}(\theta)\right| d \theta \cdot\|\varphi-\tilde{\varphi}\|_{[-r, 0]} \\
& =L_{h}\left(\left|t_{1}-t_{2}\right|+\|\varphi-\tilde{\varphi}\|_{[-r, 0]}\right),
\end{aligned}
$$

where $L_{h}=\int_{-r}^{0}\left|\gamma_{1}(\theta)\right| d \theta$. 
Moreover, from above inequality, we can see that for any bounded set $\widetilde{D} \subset C([-r, 0], X)$ and $t \in[0,1]$,

$$
\chi(h(t, \widetilde{D})) \leq \int_{-r}^{0}\left|\gamma_{1}(\theta)\right| \chi(\widetilde{D}(\theta)) d \theta \leq \int_{-r}^{0}\left|\gamma_{1}(\theta)\right| d \theta \cdot \sup _{\theta \in[-r, 0]} \chi(\widetilde{D}(\theta)) .
$$

Suppose further that there exists a constant $M^{*} \in(0,1)$ such that

$$
L_{0}+\frac{M K}{\Gamma(q+1)} \max \left\{2\|\eta\|_{L^{1}}\left\|\mu_{1}\right\|_{L^{1}}+\left\|\mu_{2}\right\|_{L^{1}}\right\}<M^{*}
$$

then (4.1) has a mild solution by Theorem 3.1.

For example, if we put

$$
\gamma_{1}(\theta)=\gamma_{2}(\theta)=e^{k \theta}, \quad q=0.5, k=7, r=1,
$$

then $L_{h}=\left(e^{7}-1\right) /\left(7 e^{7}\right) \approx 0.143, L_{g}^{*}=1 / 49 \approx 0.020, \max \left\{2\|\eta\|_{L^{1}},\left\|\mu_{1}\right\|_{L^{1}}+\left\|\mu_{2}\right\|_{L^{1}}\right\}=2\|\eta\|_{L^{1}} \approx$ $0.579, \Gamma(3 / 2) \approx 0.886, L_{0}=M L_{g}^{*}(1+\omega)+L_{h}=(1 / 49)\left(1+\left(e^{7}-1\right) /\left(7 e^{7}\right)\right)+\left(e^{7}-1\right) /\left(7 e^{7}\right) \approx 0.166$, thus, we see

$$
L_{0}+\frac{M K}{\Gamma(q+1)} \max \left\{2\|\eta\|_{L^{1}},\left\|\mu_{1}\right\|_{L^{1}}+\left\|\mu_{2}\right\|_{L^{1}}\right\}<0.9<1
$$

Example 4.2. Let $\Omega$ be a bounded domain with smooth boundary $\partial \Omega$ in $\mathbf{R}^{\mathbf{n}}$ and let $A(\xi, D)$ be the symmetric second-order differential operator given by

$$
A(\xi, D) u=-\sum_{k, l=1}^{n} \frac{\partial}{\partial \xi_{k}}\left(a_{k, l}(\xi) \frac{\partial u}{\partial \xi_{l}}\right)
$$

where $\xi=\left(\xi_{1}, \ldots, \xi_{n}\right) \in \mathbf{R}^{\mathbf{n}}$.

We assume that the coefficients $a_{k, l}(\xi)=a_{l, k}(\xi)$ are real valued and continuously differentiable in $\bar{\Omega}$ and $A(\xi, D)$ is strongly elliptic, that is, there is a constant $C_{0}>0$ such that

$$
\sum_{k, l=1}^{n} a_{k, l}(\xi) \eta_{i} \eta_{j} \geq C_{0} \sum_{k=1}^{n} \eta_{k^{\prime}}^{2}
$$

for any $\eta=\left(\eta_{1}, \ldots, \eta_{n}\right) \in \mathbf{R}^{\mathbf{n}}$.

Set $X=L^{2}(\Omega)$ and define $A$ by

$$
\begin{gathered}
D(A)=H^{2}(\Omega) \cap H_{0}^{1}(\Omega), \\
A u=A(\xi, D) u .
\end{gathered}
$$


Then, $-A$ generates an analytic semigroup $\widetilde{S}(\cdot)$ of uniformly bounded linear operators, and $\|\widetilde{S}(t)\| \leq 1[24]$.

We consider the following integrodifferential problem:

$$
\begin{gathered}
\frac{\partial^{q}}{\partial t^{q}}\left[v(t, \xi)-e^{-t} \int_{t-r}^{t} a(s-t) \sin v(s, \xi) d s\right]+A(\xi, D)\left[v(t, \xi)-e^{-t} \int_{t-r}^{t} a(s-t) \sin v(s, \xi) d s\right] \\
=\int_{0}^{t} f_{1}(s) v(s, \xi) d s+\int_{0}^{t} \int_{s-r}^{s} b(s, \tau-s) f_{2}(s, v(\tau, \xi)) d \tau d s, \quad(t, \xi) \in[0,1] \times \Omega, \\
v(t, \xi)-e^{-t} \int_{t-r}^{t} a(s-t) \sin v(s, \xi) d s=0, \quad(t, \xi) \in[0,1] \times \partial \Omega, \\
v(\theta, \xi)=v_{0}(\theta, \xi)+\int_{\Omega} \frac{c(\xi, z)}{1+|v(\theta, z)|} d z, \quad-r \leq \theta \leq 0,
\end{gathered}
$$

where $r>0, a:[-r, 0] \rightarrow \mathbf{R}, b:[0,1] \times[-r, 0] \rightarrow \mathbf{R}, v_{0}:[-r, 0] \times \Omega \rightarrow \mathbf{R}, f_{1}:[0,1] \rightarrow \mathbf{R}$ are continuous functions, $c(\xi, z) \in L^{2}(\Omega \times \Omega, \mathbf{R})$ and $\int_{-r}^{0}|a(\theta)| d \theta<1$.

For $\xi \in \Omega$ and $\varphi \in C([-r, 0], X)$, we set

$$
\begin{gathered}
x(t)(\xi)=v(t, \xi), \\
\phi(\theta)(\xi)=v_{0}(\theta, \xi), \quad \theta \in[-r, 0], \\
h(t, \varphi)(\xi)=e^{-t} \int_{-r}^{0} a(\theta) \sin \varphi(\theta)(\xi) d \theta, \\
g(\varphi(\theta))(\xi)=\int_{\Omega} \frac{c(\xi, z)}{1+|\varphi(\theta)(z)|} d z, \\
f(t, x(t), \varphi)(\xi)=f_{1}(t) x(t)(\xi)+\int_{-r}^{0} b(t, \theta) f_{2}(t, \varphi(\theta)(\xi)) d \theta .
\end{gathered}
$$

Then (4.17) can be reformulated as the abstract $(1.1)(K(t, s) \equiv 1)$.

Furthermore, we assume the following.

(1) The function $f_{2}:[0,1] \times C([-r, 0], X) \rightarrow \mathbf{R}$ is continuous, and there exist continuous functions $l(t)$ and $L_{f}(t)$ such that

$$
\begin{gathered}
\left\|f_{2}(t, \varphi)(\xi)\right\| \leq l(t)\|\varphi\|_{[-r, 0]^{\prime}} \\
\left\|f_{2}(t, \varphi)-f_{2}(t, \tilde{\varphi})\right\| \leq L_{f}(t)\|\varphi-\tilde{\varphi}\| .
\end{gathered}
$$

(2) The function $b(t, \theta)$ is continuous in $[0,1] \times[-r, 0]$ and

$$
\int_{-r}^{0}|b(t, \theta)| d \theta=p(t)<\infty
$$


(3) The function $c(\xi, z), \xi, z \in \Omega$ is measurable, and there exists a constant $\bar{N}$ such that

$$
\int_{\Omega}\|c(\xi, z)\| d z \leq \bar{N}
$$

Thus, for $t \in[0,1]$, we can see

$$
\|f(t, x(t), \varphi)\| \leq \mu_{1}(t)\|x(t)\|+\mu_{2}(t)\|\varphi\|_{[-r, 0]},
$$

where $\mu_{1}(t):=\left|f_{1}(t)\right|, \mu_{2}(t):=l(t) p(t)$.

For any bounded sets $D_{1} \subset X, D_{2} \subset C([-r, 0], X)$, we have

$$
x\left(f\left(t, D_{1}, D_{2}\right)\right) \leq \eta(t)\left(x\left(D_{1}\right)+\sup _{-r \leq \theta \leq 0} x\left(D_{2}(\theta)\right)\right), \quad \text { a.e. } t \in[0,1]
$$

where $\eta(t):=\max \left\{\left|f_{1}(t)\right|, L_{f}(t) p(t)\right\}$.

For $\varphi, \tilde{\varphi} \in C([-r, 0], X), \theta \in[-r, 0]$, we can see

$$
\|g(\varphi)(\xi)-g(\tilde{\varphi})(\xi)\| \leq\left(\int_{\Omega} \int_{\Omega} c^{2}(\xi, z) d z d \xi\right)^{1 / 2} \cdot\|\varphi-\tilde{\varphi}\|:=\widetilde{L}_{g} \cdot\|\varphi-\tilde{\varphi}\| .
$$

Moreover,

$$
\|g(\varphi)(\xi)\| \leq \int_{\Omega}\|c(\xi, z)\| d z \leq \bar{N}
$$

It is clear that

$$
\left\|h\left(t_{1}, \varphi\right)-h\left(t_{2}, \tilde{\varphi}\right)\right\| \leq \bar{L}_{h} \cdot\left(\left|t_{1}-t_{2}\right|+\|\varphi-\tilde{\varphi}\|_{[-r, 0]}\right),
$$

where $\bar{L}_{h}:=\max \left\{1,(\text { meas } \cdot(\Omega))^{1 / 2}\right\} \cdot \int_{-r}^{0}|a(\theta)| d \theta$, and for any bounded set $\tilde{D} \subset C([-r, 0], X)$ and $t \in[0,1]$,

$$
x(h(t, \tilde{D})) \leq \int_{-r}^{0}|a(\theta)| d \theta \cdot \sup _{\theta \in[-r, 0]} x(\tilde{D}(\theta)) .
$$

Suppose further that there exists a constant $\widetilde{M}^{*} \in(0,1)$ such that

$$
L_{0}+\frac{1}{\Gamma(q+1)} \max \left\{2\|\eta\|_{L^{1}}\left\|\mu_{1}\right\|_{L^{1}}+\left\|\mu_{2}\right\|_{L^{1}}\right\}<\widetilde{M}^{*}
$$

where $L_{0}=\widetilde{L}_{g}\left(1+\int_{-r}^{0}|a(\theta)| d \theta\right)+\bar{L}_{h}$, then (4.17) has a mild solution by Theorem 3.1. 


\section{Acknowledgments}

The authors are grateful to the referees for their valuable suggestions. The first author is supported by the NSF of Yunnan Province (2009ZC054M).

\section{References}

[1] M. M. El-Borai, "Semigroups and some nonlinear fractional differential equations," Applied Mathematics and Computation, vol. 149, no. 3, pp. 823-831, 2004.

[2] M. M. El-Borai, "Some probability densities and fundamental solutions of fractional evolution equations," Chaos, Solitons and Fractals, vol. 14, no. 3, pp. 433-440, 2002.

[3] F. Li, "Solvability of nonautonomous fractional integrodifferential equations with infinite delay," Advances in Difference Equations, vol. 2011, Article ID 806729, 18 pages, 2011.

[4] F. Li, "An existence result for fractional differential equations of neutral type with infinite delay," Electronic Journal of Qualitative Theory of Differential Equations, no. 52, pp. 1-15, 2011.

[5] G. M. Mophou and G. M. N'Guérékata, "Existence of the mild solution for some fractional differential equations with nonlocal conditions," Semigroup Forum, vol. 79, no. 2, pp. 315-322, 2009.

[6] G. M. Mophou and G. M. N'Guérékata, "Existence of mild solutions of some semilinear neutral fractional functional evolution equations with infinite delay," Applied Mathematics and Computation, vol. 216, no. 1, pp. 61-69, 2010.

[7] G. M. Mophou and G. M. N'Guérékata, "A note on a semilinear fractional differential equation of neutral type with infinite delay," Advances in Difference Equations, vol. 2010, Article ID 674630, 8 pages, 2010.

[8] L. Hu, Y. Ren, and R. Sakthivel, "Existence and uniqueness of mild solutions for semilinear integrodifferential equations of fractional order with nonlocal initial conditions and delays," Semigroup Forum, vol. 79, no. 3, pp. 507-514, 2009.

[9] Y. Ren, Y. Qin, and R. Sakthivel, "Existence results for fractional order semilinear integro-differential evolution equations with infinite delay," Integral Equations and Operator Theory, vol. 67, no. 1, pp. 3349, 2010.

[10] G. M. N'Guérékata, "A Cauchy problem for some fractional abstract differential equation with non local conditions," Nonlinear Analysis. Theory, Methods E Applications, vol. 70, no. 5, pp. 1873-1876, 2009.

[11] V. Obukhovskii and J.-C. Yao, "Some existence results for fractional functional differential equations," Fixed Point Theory, vol. 11, no. 1, pp. 85-96, 2010.

[12] T.-J. Xiao, J. Liang, and J. van Casteren, "Time dependent Desch-Schappacher type perturbations of Volterra integral equations," Integral Equations and Operator Theory, vol. 44, no. 4, pp. 494-506, 2002.

[13] J. Liang and T.-J. Xiao, "Solvability of the Cauchy problem for infinite delay equations," Nonlinear Analysis. Theory, Methods \& Applications, vol. 58, no. 3-4, pp. 271-297, 2004.

[14] T.-J. Xiao and J. Liang, "Blow-up and global existence of solutions to integral equations with infinite delay in Banach spaces," Nonlinear Analysis. Theory, Methods \& Applications, vol. 71, no. 12, pp. e1442e1447, 2009.

[15] M. Benchohra and S. K. Ntouyas, "Nonlocal Cauchy problems for neutral functional differential and integrodifferential inclusions in Banach spaces," Journal of Mathematical Analysis and Applications, vol. 258, no. 2, pp. 573-590, 2001.

[16] J. Liang, J. H. Liu, and T.-J. Xiao, “Nonlocal impulsive problems for nonlinear differential equations in Banach spaces," Mathematical and Computer Modelling, vol. 49, no. 3-4, pp. 798-804, 2009.

[17] J. Liang, J. Liu, and T.-J. Xiao, "Nonlocal Cauchy problems governed by compact operator families," Nonlinear Analysis. Theory, Methods E Applications, vol. 57, no. 2, pp. 183-189, 2004.

[18] J. Liang and T.-J. Xiao, "Semilinear integrodifferential equations with nonlocal initial conditions," Computers \& Mathematics with Applications, vol. 47, no. 6-7, pp. 863-875, 2004.

[19] T.-J. Xiao and J. Liang, "Existence of classical solutions to nonautonomous nonlocal parabolic problems," Nonlinear Analysis, Theory, Methods and Applications, vol. 63, no. 5-7, pp. e225-e232, 2005.

[20] R. R. Akhmerov, M. I. Kamenskiŭ, A. S. Potapov, A. E. Rodkina, and B. N. Sadovskiŭ, Measures of Noncompactness and Condensing Operators, Birkhäuser, Boston-Basel, Berlin, Germany, 1992.

[21] M. Kamenskii, V. Obukhovskii, and P. Zecca, Condensing Multivalued Maps and Semilinear Differential Inclusions in Banach Spaces, vol. 7 of de Gruyter Series in Nonlinear Analysis and Applications, Walter de Gruyte, Berlin, Germany, 2001.

[22] D. Henry, Geometric Theory of Semilinear Parabolic Partial Differential Equations, Springer, Berlin, Germany, 1989. 
[23] F. Mainardi, P. Paradisi, and R. Gorenflo, "Probability distributions generated by fractional diffusion equations," in Econophysics: An Emerging Science, J. Kertesz and I. Kondor, Eds., Kluwer Academic, Dodrecht, The Netherlands, 2000.

[24] A. Pazy, Semigroups of Linear Operators and Applications to Partial Differential Equations, vol. 44 of Applied Mathematical Sciences, Springer, New York, NY, USA, 1983. 


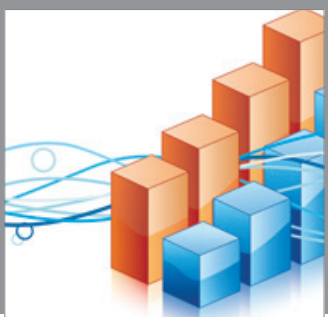

Advances in

Operations Research

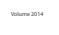

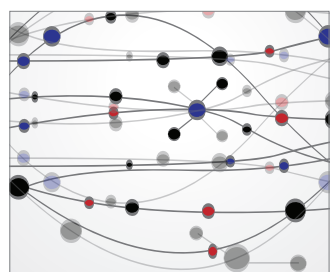

\section{The Scientific} World Journal
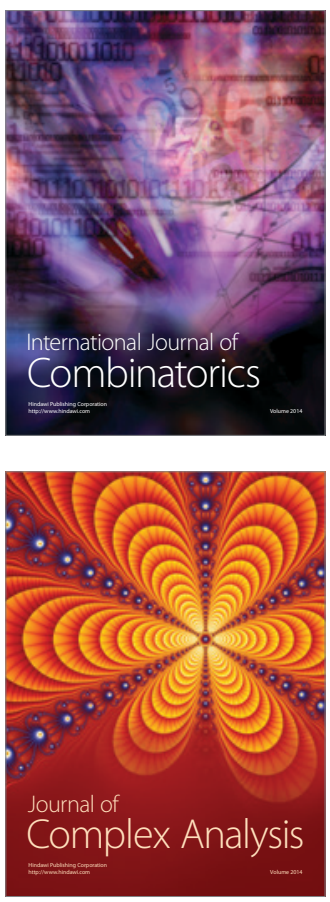

International Journal of

Mathematics and

Mathematical

Sciences
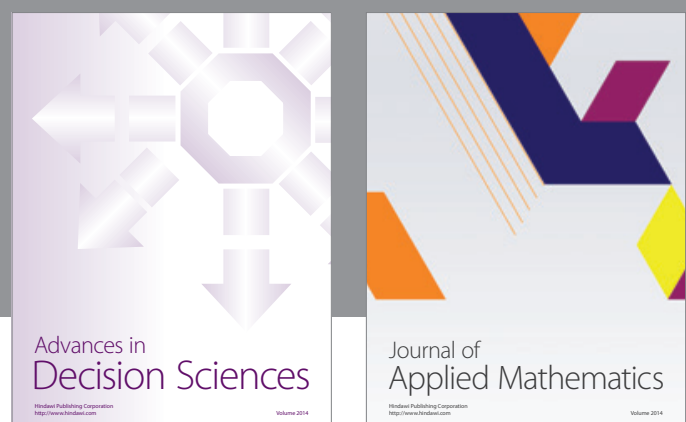

Journal of

Applied Mathematics
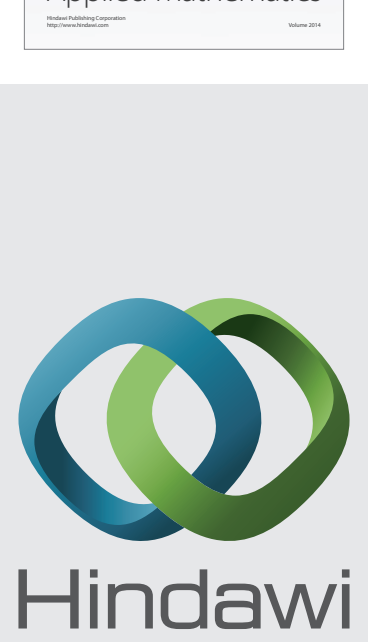

Submit your manuscripts at http://www.hindawi.com
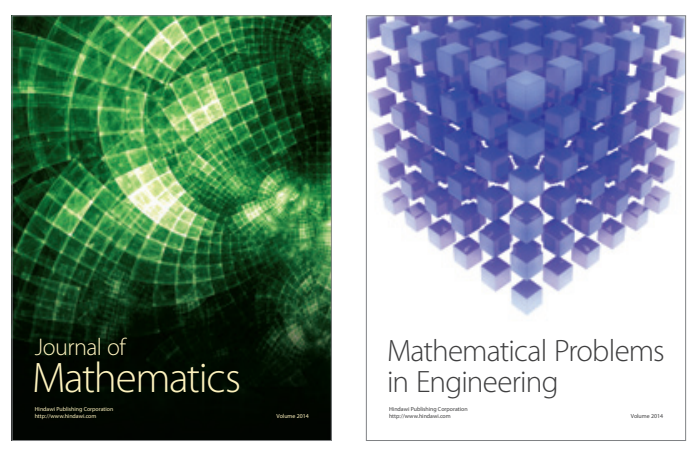

Mathematical Problems in Engineering
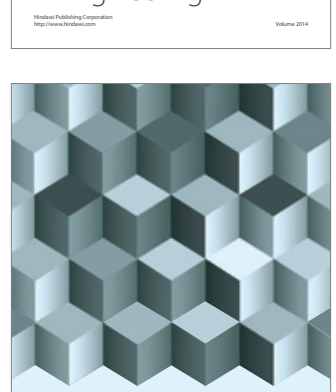

Journal of

Function Spaces
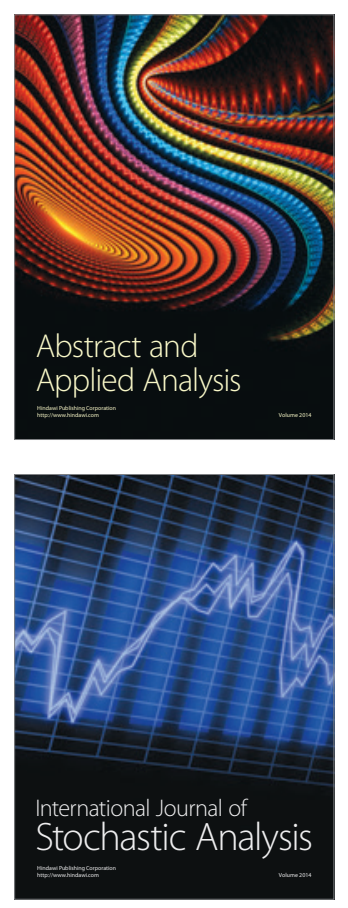

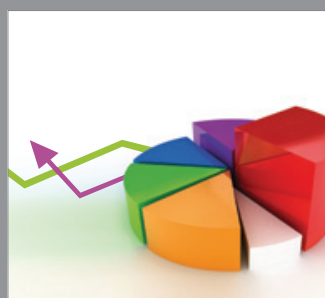

ournal of

Probability and Statistics

Promensencen
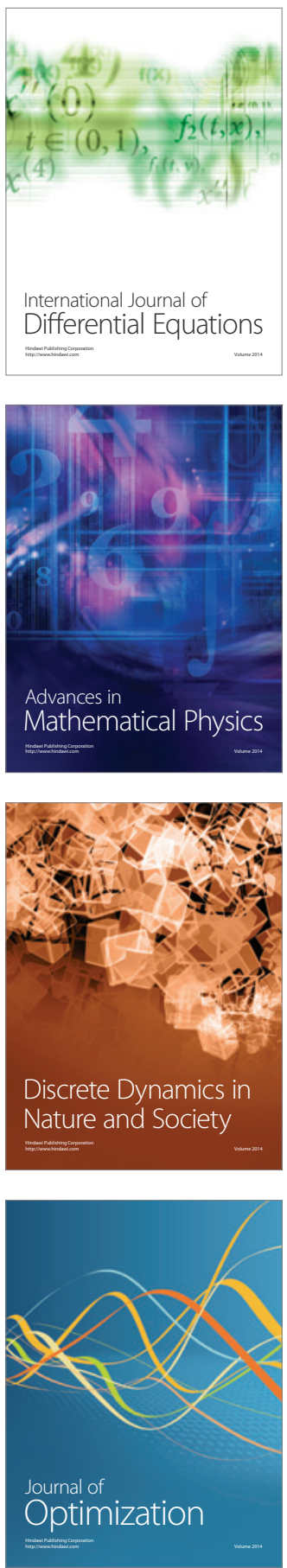